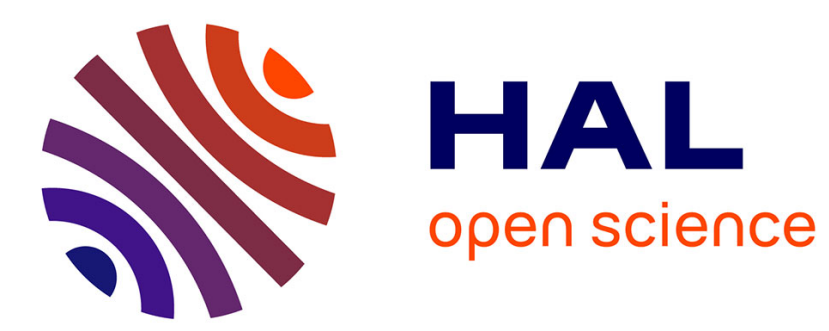

\title{
ORDRE MAGNÉTIQUE A DEUX DIMENSIONS DANS Ca2MnO4
}

\author{
G. Ollivier, G. Buisson
}

\section{To cite this version:}

G. Ollivier, G. Buisson. ORDRE MAGNÉTIQUE A DEUX DIMENSIONS DANS Ca2MnO4. Journal de Physique Colloques, 1971, 32 (C5), pp.C5a-189-C5a-189. 10.1051/jphyscol:1971524 . jpa00214744

\section{HAL Id: jpa-00214744 https://hal.science/jpa-00214744}

Submitted on 1 Jan 1971

HAL is a multi-disciplinary open access archive for the deposit and dissemination of scientific research documents, whether they are published or not. The documents may come from teaching and research institutions in France or abroad, or from public or private research centers.
L'archive ouverte pluridisciplinaire HAL, est destinée au dépôt et à la diffusion de documents scientifiques de niveau recherche, publiés ou non, émanant des établissements d'enseignement et de recherche français ou étrangers, des laboratoires publics ou privés. 


\title{
ORDRE MAGNÉTIQUE A DEUX DIMENSIONS DANS $\mathrm{Ca}_{2} \mathrm{MnO}_{4}$
}

\author{
G. OLLIVIER et G. BUISSON \\ Laboratoire de Diffraction neutronique, CEN Grenoble, Cedex 85, 38, Grenoble-gare \\ Paru dans Solid State Communications, 1971, 9, 235-240.

\section{COMPLÉMENTS BIBLIOGRAPHIQUES \\ SUR LA MISE EN ÉVIDENCE EXPÉRIMENTALE \\ D'UN ORDRE MAGNÉTIQUE \\ DANS LES SYSTËMES ANTIFERROMAGNÉTIQUES} \\ A UNE ET DEUX DIMENSIONS
}

\author{
G. PARETTE
}

Service de Physique du Solide et de Résonance Magnétique

Centre d'Etudes Nucléaires de Saclay, BP nº 2, 91, Gif-sur-Yvette, France

La communication de B. Jancovici [1] montre que théoriquement il n'est pas prévu à température non nulle de transition ordre-désordre dans les systèmes magnétiques à une et deux dimensions présentant les caractères suivants :

1. Hamiltonien d'Heisenberg avec interactions d'échange à courte portée.

2. «Volume» infini. - En d'autres termes on ne peut définir pour de tels systèmes un ordre à grande distance. Cependant de nombreuses expériences de diffusion de neutrons ont mis en évidence dans un certain nombre de substances magnétiques sinon un ordre à grande distance, du moins des corrélations entre spins s'étendant à des distances grandes comparées aux distances interatomiques. Il ne nous semble pas que les résultats expérimentaux soient en désaccord avec les prévisions théoriques compte tenu de ce que la nature à trois dimensions ne peut offrir que des exemples imparfaits de systèmes à une et deux dimensions: les substances étudiées présentent toujours quelque anisotropie qui, si petite soit-elle, fait que l'hamiltonien n'est jamais rigoureusement un hamiltonien d'Heisenberg. D'autre part même s'il était possible de trouver un système idéal, le « volume » d'un tel système dans la réalité est toujours fini.
Enfin le caractère trimensionnel de la substance (qui finit toujours par s'imposer) perturbe sérieusement l'observation d'un ordre ou d'un "quasi-ordre " à une ou deux dimensions.

Toutes ces raisons amènent à modifier les conclusions de la théorie [1].

Nous n'avons pas la prétention de présenter une liste exhaustive des études qui ont été faites sur les systèmes réels à une et deux dimensions par diffusion de neutrons. Parmi les travaux les plus significatifs, citons ceux qui ont été effectués sur le réacteur à haut flux du Brookhaven National Laboratory (HFBR) et au CEN-G. Les travaux réalisés à Grenoble ont fait l'objet de la précédente communication.

Les systèmes magnétiques présentant un caractère bidimensionnel marqué ont été étudiés les premiers, citons-en quelques-uns :

$$
\begin{aligned}
& \mathrm{K}_{2} \mathrm{NiF}_{4} \text { [2], [3], [4], [5] } \\
& \quad \mathrm{Mn}(\mathrm{HCOO})_{2}, 2 \mathrm{H}_{2} \mathrm{O} \text { [6], } \mathrm{Ca}_{2} \mathrm{MnO}_{4} \text { [7], [8] . }
\end{aligned}
$$

En ce qui concerne les exemples de systèmes magnétiques unidimensionnels que peut fournir la nature, citons :

$$
\begin{array}{ll} 
& 2 \mathrm{H}_{2} \mathrm{O}[9] \\
\mathrm{CsMnCl}_{3}, & 2 \mathrm{D}_{2} \mathrm{O}[10], \quad\left(\mathrm{CD}_{3}\right)_{4} \mathrm{NMnCl}_{3}[11] .
\end{array}
$$

